\title{
Computation of Fresnel Integrals. II
}

\section{Klaus D. Mielenz}

Oakland, MD 21550

kmielenz@mail.gcnet.net
This paper describes an improved method for computing Fresnel integrals with an error of less than $1 \times 10^{-9}$. The method is based on a known approximate formula for a different integral which is due to Boersma and referenced by Abramowitz and Stegun.
Key words: computation; Fresnel integrals; rational approximations; series expansions.

Accepted: July 17, 2000

Available online: http://www.nist.gov/jres

\section{Improved Computation of Fresnel Integrals}

In a previous paper [1], this author presented formulas for numerical computations of the Fresnel cosine and sine integrals,

$$
\begin{aligned}
C(x) & =\int_{0}^{x} \mathrm{~d} t \cos \left(\frac{\pi}{2} t^{2}\right) \\
& =\frac{1}{2}+f(x) \sin \left(\frac{\pi}{2} x^{2}\right)-g(x) \cos \left(\frac{\pi}{2} x^{2}\right), \\
S(x) & =\int_{0}^{x} \mathrm{~d} t \sin \left(\frac{\pi}{2} t^{2}\right) \\
& =\frac{1}{2}-f(x) \cos \left(\frac{\pi}{2} x^{2}\right)-g(x) \sin \left(\frac{\pi}{2} x^{2}\right),
\end{aligned}
$$

to six significant figures by using the first three terms of the Taylor expansions of $C(x)$ and $S(x)$ for $|x| \leq 0.6$, the first three terms of the asymptotic expansions of the auxiliary functions $f(x)$ and $g(x)$ for $|x| \geq 3$, and modified rational approximations of $f(x)$ and $g(x)$ for the mid range. These formulas proved hard to use because they involve too many numerical constants.

It was found subsequently that a simpler and more accurate method of computation can be based on a formula derived by Boersma [2],

$$
\begin{gathered}
\int_{0}^{u} \frac{\mathrm{d} \tau \mathrm{e}^{-\mathrm{i} \tau}}{\sqrt{2 \pi \tau}}=\frac{1-\mathrm{i}}{2}+\mathrm{e}^{-\mathrm{i} u} \sqrt{\frac{4}{u}} \sum_{n=0}^{11}\left(p_{n}+\mathrm{i} q_{n}\right)\left(\frac{4}{u}\right)^{n}, \\
u \geq 4,
\end{gathered}
$$

where $p_{n}$ and $q_{n}$ are numerical constants tabulated in Ref. [2] and the notation has been changed in order to avoid confusion with symbols used elsewhere in this paper. On substituting $t=\pi \tau^{2} / 2$ and $x=\pi u^{2} / 2$ this is transformed into

$$
\begin{aligned}
& \int_{0}^{x} \mathrm{~d} t \mathrm{e}^{-\mathrm{i} \pi t^{2} / 2}=C(x)-\mathrm{i} S(x)=\frac{1-\mathrm{i}}{2} \\
& -\mathrm{e}^{-\mathrm{i} \pi x^{2} / 2} \sum_{n=0}^{11} \frac{g_{n}-\mathrm{i} f_{n}}{x^{2 n+1}}, \quad x \geq 1.6,
\end{aligned}
$$




$$
f_{n}=(8 / \pi)^{n+1 / 2} q_{n}, \quad g_{n}=-(8 / \pi)^{n+1 / 2} p_{n}
$$

Hence it follows by separation of real and imaginary parts and comparison with Eqs. (1a) and (1b) that

$$
f(x)=\sum_{n=0}^{11} f_{n} x^{-2 n-1}, \quad g(x)=\sum_{n=0}^{11} g_{n} x^{-2 n-1} .
$$

The required coefficients, as computed from Boersma's data and Eq. (2c), are listed in Table 1. Equation (2d) was tested by computing sample values of $C(x)$ and $S(x)$ and comparing them to the values tabulated in Ref. [3] to eight digits. The agreement was perfect, which is consistent with Boersma's statement that the error of Eq. (2a) is less than $5 \times 10^{-10}$.

Table 1. Numerical values of $f_{n}$ and $g_{n}$

\begin{tabular}{rcc}
\hline \hline$n$ & $f_{n}$ & \multicolumn{1}{c}{$g_{n}$} \\
\hline 0 & 0.318309844 & 0 \\
1 & $9.34626 \mathrm{E}-08$ & 0.101321519 \\
2 & -0.09676631 & $-4.07292 \mathrm{E}-05$ \\
3 & 0.000606222 & -0.152068115 \\
4 & 0.325539361 & -0.046292605 \\
5 & 0.325206461 & 1.622793598 \\
6 & -7.450551455 & -5.199186089 \\
7 & 32.20380908 & 7.477942354 \\
8 & -78.8035274 & -0.695291507 \\
9 & 118.5343352 & -15.10996796 \\
10 & -102.4339798 & 22.28401942 \\
11 & 39.06207702 & -10.89968491 \\
\end{tabular}

The above method is implicitly contained in Ref. [3], which mentioned Boersma's paper as well as the relationship between the integrals in Eqs. (1a), (1b), and (2a). Boersma also gave an approximation formula similar to Eq. (2a) for $|u| \leq 4$, or $|x| \leq 1$.6. This was not used in this work because in this range it is simpler to compute $C(x)$ and $S(x)$ by using their Taylor expansions [3],

$$
\begin{gathered}
C(x)=\sum_{n=0}^{\infty} c_{n} x^{4 n+1}, \quad c_{0}=1, \\
c_{n+1}=\frac{-\pi^{2}(4 n+1) c_{n}}{4(2 n+1)(2 n+2)(4 n+5)}, \\
S(x)=\sum_{n=0}^{\infty} s_{n} x^{4 n+3}, \quad s_{0}=\frac{\pi}{6}, \\
s_{n+1}=\frac{-\pi^{2}(4 n+3) s_{n}}{4(2 n+2)(2 n+3)(4 n+7)} .
\end{gathered}
$$

These give results with errors less than $6 \times 10^{-10}$ for $|x| \leq 1.6$ if the first 11 terms of the expansions are carried.

Software and algorithms for computing Fresnel integrals in Fortran and C (not based on this paper) are also available on the Internet $[4,5]$.

\section{References}

[1] K. D. Mielenz, J. Res. Natl. Inst. Stand. Technol. 102, 363 (1997).

[2] J. Boersma, Math. Computation 14, 380 (1960).

[3] M. Abramowitz and I. A. Stegun, eds., Handbook of Mathematical Functions, (U.S. Gov. Printing Office, Washington, DC, 1972).

[4] D. W. Lozier and F. W. J. Olver, Numerical Evaluation of Special Functions in Mathematics of Computation, 1943-1993: A Half Century of Computational Mathematics, W. Gautschi, ed., Am. Math. Soc. (1994) pp. 79-125. Also available at http:// math.nist.gov/nesf.

[5] R. F. Boisvert, The Architecture of an Intelligent Virtual Mathematical Software Repository System, Mathematics and Computers in Simulation, Vol. 36 (1994) pp. 269-279. Also available at http://math.nist.gov/gams.

About the author: Klaus D. Mielenz is a physicist and retired Chief of the Radiometric Physics Division of the NIST Physics Laboratory. The National Institute of Standards and Technology is an agency of the Technology Administration, U.S. Department of Commerce. 